\title{
Use of Gold Nanoparticles in Diagnostics, Surgery and Medicine: A Review
}

\author{
ASM Giasuddin ${ }^{1}$, KA Jhuma ${ }^{2}$, AM Mujibul Haq ${ }^{3}$ \\ ${ }^{1}$ Dept of Medical Laboratory Science State College of Health Sciences, Dhaka \\ ${ }^{2}$ Dept of Biochemistry, Medical College for Women and Hospital, Dhaka \\ ${ }^{3}$ Dept of Medicine, Medical College for Women and Hospital, Dhaka
}

ABSTRACT

\begin{abstract}
Noble metals and their compounds have a long and distinguished history as therapeutic agents in medicine. Recent years have seen tremendous progress in the design and study of nanomaterials geared towards biological and biomedical applications. Particularly gold nanoparticles have attracted intensive interest, because they are easily prepared, have low toxicity and can be readily attached to molecules of biological interest. The gold nanoparticles have become more precious than pretty gold because of their wide use and applications. The present article gave a critical review of the wide applications and uses of gold nanoparticles in diagnostics, surgery and medicine.
\end{abstract}

Key words: Gold, Nanoparticle, Diagnostic

\section{Introduction}

Recent years have seen tremendous progress in the design and study of nanomaterials geared towards biological and biomedical applications ${ }^{1}$. Noble metal nanostructures attract much interest because of their unique properties, including large optical field enhancements resulting in the strong scattering and absorption of light. The enhancement in the optical and photothermal properties of noble metal nanoparticles arises from resonant oscillation of their free electrons in the presence of light, also known as localised surface plasmon resonance $(\mathrm{LSPR})^{2}$. The plasmon resonance, i.e. LSPR, can either radiate light (Mie Scattering), a process that finds great utility in optical and imaging fields, or be rapidly converted to heat (absorption); the later mechanism of dissipation has opened up applications in several new areas ${ }^{2}$. The ability to integrate metal nanoparticles into biological systems with general lack of toxicity has had greatest impact in biology and medicine. Among noble metal particles, gold nanoparticles have attracted intensive interest because they are easily prepared, have low toxicity and can be readily attached to molecules of biological interest ${ }^{3}$.The present article gave a critical

Bangladesh J Med Biochem 2012; 5(2): 56-60 review of the wide spread applications and uses of gold nanoparticles in diagnostics, surgery and medicine.

\section{Gold Nanoparticles}

Noble metal nanoparticles, particularly gold colloidal nanoparticles, were chemically synthesized with different passivating ligands including didecylsulfides, citrate and magnesium oleate molecules ${ }^{3-6}$. From the analysis of high resolution transmission electron microscopy (HRTEM) photomicrographs of the nanoparticles, the average diameter of gold nanoparticles passivated with didecylsulfide and citrate and magnesium oleate-stabilised gold nanoparticles were 5.30 .8 and $15.01 .7 \mathrm{~nm}^{2}$ respectively ${ }^{3}$. The position, shape and magnitude of the plasmon absorption band of colloidal solutions of nanoparticles depend strongly on several factors - the particle size, the metal, the refractive index of the solvent used, the surface-absorbed species, the temperature of the colloidal solution and the degree of aggregation $3,7,8$.

Thus, chemical synthesis offers a versatile, cost effective approach, and has been used broadly to 
prepare gold nanoparticles with various passivating ligands. However, gas-phase growth provides a beneficial alternative to create nanoparticle arrays. In particular, size-selected nanoclusters with narrow size distribution (5\%) can be produced and deposited into a variety of supports, creating the potential for the investigation of single nanoparticle fluorescence in dilute arrays. A state-of-the-art magnetron sputtering gas-aggregation cluster source, which incorporated a novel time-of-flight mass selector, has been used to produce Au5800 nanoclusters with size distribution of $5 \% 3,9$. The HRTEM image of Au5800 cluster reveals monodispersed nanoparticles, mostly of special shape, deposited on amorphous carbon film support $^{3}$. The statistical analysis of the nanoparticle shapes provides a distribution of particles as function of aspect ratio. Twenty seven percent of analysed particles have an aspect ratio of 1 , implying a spherical or almost spherical shape. Sixty one present of particles have close to spherical shapes with an aspect ratio 1.550 .4 . Twelve percent of particles have an elongated shape with an aspect ration larger than 2. With careful control over the cluster formation condition, one can adjust this shape distribution and enhance, for instance, the yield of elongated nanoclusters ${ }^{3,9}$.

\section{Biomedical Uses \& Applications}

Precious metals and their compounds as therapeutic agents, particularly of gold, have a long and distinguish history in medicine. The use of gold in medicine have evolved over thousands of years ${ }^{10,11}$. In China, gold was used in the treatment of ailments such as smallpox, skin ulcers and measles. In Japan, thin gold foils placed in tea, sake and food were seen as beneficial to health. In Bangladesh-PakistanIndia, traditional Ayurvedic medicines are still used widely with gold taken as a 'rejuvenator' by millions of people each year. A typical daily dose includes 1-2mg of gold incorporated into a mixture of herbs ${ }^{11}$. As medical science and medicine have advanced, so too the biomedical uses and applications of gold. Over the years, the gold nanoparticles have become more precious than pretty gold. Most important reason for this has been their wide uses and applications in the fields of diagnostics, surgery and medicine ${ }^{11,12,13}$. The ability to integrate metal, particularly gold and silver, nanoparticles into biological systems has had greatest impact in biology \& medicine. Some investigators reported the widespread use of gold nanoparticles in four areas of biology, i.e. labeling, delivering, heating and sensing2,14. Most applications are largely related to the excellent biocompatibility and innertness of gold as a material and resistance to bacterial infection.

\section{In Surgery}

A resistance to bacterial infection has led to a long tradition of gold being used in microsurgery of the ear and other procedures which require implants that are at risk of infection, including in the eye. In addition to gold's resistance to bacteria, the radiopacity of the metal means that gold-plated stents offer the best visibility under X-ray, enabling them to be positioned accurately during surgery. In 2001, Boston Scientific produced the Niroyal stent, one of the first gold-plated stents, largely in response to the need for stents that could be placed more accurately. The biological inertness of gold was found to be important in this application and goldplated stents have been found to produce the least number of macroscopic changes in surrounding intravascular tissue (Figure-1) (11,12. $^{3}$

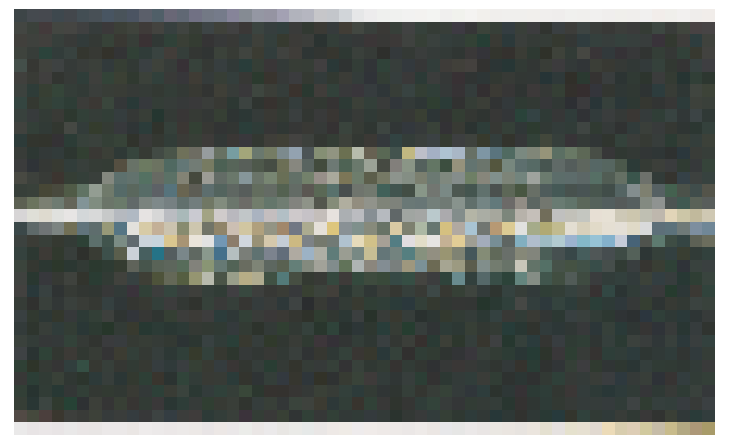

Figure-1: The radiopacity of the metal means that gold-plated stents offer the best visibility under X-ray, enabling them to be positioned accurately during surgery.

A new surgical procedure for prostate cancer involves the insertion of three gold grains into the prostate. The position of the gold grains can 
be detected using X-rays, allowing doctors to target the position of the prostate within one or two millimeters and thereby allow a more precise dose of radiation to be administered to a more targeted area for the treatment of the tumour. While this is a relatively new application, radioactive gold was used in some of the very first treatments of cancer dating back to $1955^{1,2,13}$.

\section{In Medicine}

More recently, new technologies have used the ability of tiny gold nanoparticles to collect specifically in a cancerous tumour by passing through the inherently leaky blood vessels attached to a tumour. Thus, when injected into a patient, there is a means by which a potent anticancer compound attached to a gold nanoparticle can be delivered directly and accurately to a tumour while avoiding surrounding healthy tissues. Such an effective drug delivery mechanism with reduced toxicity is considered to be a major step forward in cancer treatment, limiting side effects such as reduced immunity and hair loss ${ }^{11,15,16}$.

CystImmune Sciences, USA has used this strategy to engineer new colloidal gold based drug compounds that harness the therapeutic potential of potent anticancer agents. CytImmmune's lead drug candidate, Aurimune, (CYT-6091) consists of recombinant human tumor necrosis factor alpha ("TNF- ") a well documented and widely accepted tumor-killing agent, bound to the surface of pegylated colloidal gold nanoparticles. The mechanism underlying CytImmune's tumor-targeted delivery of TNF- is attributable to the size and composition of the nanoparticles-regardless of tumor type. By simultaneously binding TNFand PEG-Thiol to the surface of colloidal gold nanoparticles, the therapeutic payload travels safely through the blood stream avoiding immune detection and is preferentially delivered to the site of disease. At 27 nanometers in size, Aurimmune primarily and preferentially exits the circulation through leaky, newly formed vasculature at tumor sites, selectively passing through gaps in blood vessel walls (fenestrations) ${ }^{15,16}$.
Phase 1 clinical data demonstrate that: (i) Aurimune (CYT-6091) safely and systemically delivered TNF- in humans far beyond concentrations attained in previous human studies; (ii) the fever side effect was easily managed and separated from hypotension (the dose limiting effect of TNF- ); and (iii) based on tissue biopsies from treated patients, Aurimune accumulates in and around tumor sites, avoiding uptake by liver, spleen and healthy tissues and immune system detection.

The Aurimmune Phase 2 clinical programme is designed to mimic the isolated limb perfusion (ILP) protocol - combining Aurimune with a chemotherapy - and will include treating pancreatic cancer, melanoma, soft tissue sarcoma, ovarian and breast cancer patients with Aurimune followed by standard of care chemotherapy for each indication. If successful, these Phase 2 trials will not only confirm Aurimune's broad-based versatility and therapeutic value of TNF-, but also create an accelerated commercialization pathway ${ }^{15,16}$.

TAXOL ${ }^{\circledR}$ (paclitaxel) Injection (www.rxlist. com/taxoldrug.htm ): TAXOL (Paclitaxel), a natural product with antitumor activity, is obtained via a semi-synthetic process from Taxus baccata17. The chemical name for paclitaxel is 5 20-Epoxy-1,2, 4, 7, 10, 13 hexahydroxytax-11-en-9-one 4,10-diacetate 2benzoate 13-ester with (2R,3S)-N-benzoyl-3phenylisoserine. Paclitaxel is a white to offwhite crystalline powder with the empirical formula $\mathrm{C}_{47} \mathrm{H}_{51} \mathrm{NO}_{14}$ and a molecular weight of 853.9. It is highly lipophilic, insoluble in water, and melts at around $216-217^{\circ} \mathrm{C}$. TAXOL is indicated as first-line and subsequent therapy for the treatment of advanced carcinoma of the ovary. As first-line therapy, TAXOL is indicated in combination with cisplatin. TAXOL is indicated for the adjuvant treatment of nodepositive breast cancer administered sequentially to standard doxorubicin-containing combination chemotherapy. In the clinical trial, there was an overall favorable effect on disease-free and overall survival in the total population of patients with receptor-positive and receptor- 
negative tumors, but the benefit has been specifically demonstrated by available data (median follow-up 30 months) only in the patients with estrogen and progesterone receptor-negative tumors. TAXOL is indicated for the treatment of breast cancer after failure of combination chemotherapy for metastatic disease or relapse within 6 months of adjuvant chemotherapy. Prior therapy should have included in patients an anthracycline unless cotraindicated. Taxol, in combination with cisplatin, is indicated for the first-line treatment of non-small cell lung cancer patients who are not candidates for potentially curative surgery and/or radiation therapy. TAXOL is indicated for the second-line treatment of AIDS-related Kaposi's sarcoma. TAXOL is a cytotoxic anticancer drug and, as with other potentially toxic compounds, caution should be exercised in handling TAXOL ${ }^{17}$.

This tumour targeting technology has also been used by Rice University chemists, who have developed a way to load dozens of molecules of the anticancer drug paclitaxel (Taxol) onto tiny gold spheres. The result is a tiny ball, many times smaller than a living cell, that literally bristles with the drug (Figure-2) ${ }^{11,17}$. Similar developments have also been used in advancing the treatment of human immunodeficiency virus (HIV) infection. Researchers at North Carolina State University discovered that tiny gold particles added to a previously failed drug against HIV could rekindle its ability to stop the virus invading the body's immune system.

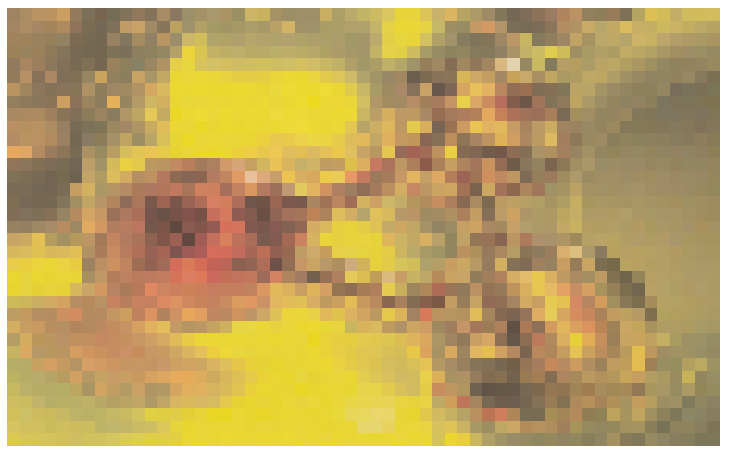

Figure-2: A new delivery system for the anticancer drug Taxol centres on a tiny ball comprising gold molecules that is barely wider than a strand of DNA.
Another US company is developing a therapy using gold nanoshells (tiny particles of gold wrapped around silica) that, once injected into the body, accumulate in cancerous tumour. Rather than being used as a means of drug delivery, these gold nanoshells convert absorbed light from a laser into heat, which then destroys the cancerous cells that form the tumour. The company is hoping to commence human trials of the treatment using patients with head and neck cancer ${ }^{11,18,19}$.

\section{Conclusions}

Although the history of chrysotherapy, i.e. use of gold compounds in medicine, is long and distinguished, work after the Second World War demonstrated conclusively that gold drugs are effective in treating patients with rheumatoid arthritis. Many experts still consider gold to be among the most effective drugs for reducing inflammation in joints, thus reducing the symptoms of pain and stiffness. Several forms of gold are available, but usually one of the injectable forms of gold salt (e.g. gold sodium thiomalate [Myochrysine] or aurothioglucose [Solganal] is used. An oral tablet containing gold, called auranofin (Radaura), is also available. As diseases evolve, so too does the need for this robust and versatile metal to aid in the treatment of a range of conditions, now and into the future. That is why gold nanoparticles are medically more precious than pretty gold. Currently, the World Gold Council's Gold Research Opportunities Worldwide (GROW) programme supports research into new uses for the precious metal 'gold'.

\section{References}

1. Huang X, Jain PK, El-Sayed IH, E1-Sayed MA. Gold nanoparticles: Interesting optical properties and recent applications in cancer diagnostics and therapy. Nanomed 2007; 2: 681-93.

2. Jain PK, Huang X, El-Sayed IH, E1-Sayed MA. Noble metals on the nanoscale: opticaland photothermal properties and some applications in imaging, sensing, biology and medicine. Acc Chem Res 2008 May; [Epub ahead of print]

3. Chen Yu, Preece JA, Palmer RE. Processing and characterisation of gold nanoparticles for use in plasmon probe spectroscopy and microscopy of 
biosystems. Ann NY Acad Sci 2008; 1130: 201-206 (doi: 10.1196/annals. 1430.051).

4. Shelley EJ, Ryan D, Johnson SR, et al. Dialkyl sulfides: novel passivating agents for gold nanoparticles. Langmuir 2002; 18: 1791-95.

5. Frens G. Controlled nucleation for regulation of particle-size in monodisperse gold suspension. Nat Phys Sci 1973; 241:20-22.

6. HE HX, Zhang H, Li QG, et al. Fabrication of designed architectures of Au nanoparticles on solid substrate with printed self-assembled monolayers as templates. Langmuir 2000; 16: 3846-51.

7. Daniel MC, Astruc D. Gold nanoparticles: assembly, supramolecular chemistry, quantum-size-related properties, and applications toward biology, catalysis, and nanotechnology. Chem Rev 2004; 104: 293-346.

8. Thomas, KG, Kamat PV. Making gold nanoparticles glow: enhanced emission from a surface-bound fluoroprobe. J Am Chem Soc 2000; 122: 2655-56.

9. Palmer RE, Pratontep S, Boyen HG. Nanostructured surfaces from size-selected clusters. Nat Mater 2003; 2: 443-48.

10. Jennings T, Strouse G. Past, present and future of gold nanoparticles. Adv Exp Med Biol 2007; 620: 34-47.

11. Holiday R. Use of gold in medicine and surgery. Biomedical Scientist (The Official Gazette of the Institute of Biomedical science, UK) 2008; 962-63.
12. Tanigawa W, Sawada S, Kobayah M. Reaction of the aortic wall to six metallic stent materials. Acad Radiol 1995; 2: 379-84.

13. Wheeler HB, Jaques WE, Botsford TW. Experiences with the use of radioactive colloidal gold in the treatment of cancer. Ann Surg 1955; 141: 208-17.

14. Sperling RA, Gil PR, Zhang F, Zanella M, Parak WJ. Biological applications of gold nanoparticles. Chem Soc Rev 2008; 37: 1896-1908 (doi: 10.1039/ b712170a).

15. Tamarkin L, Myer L, Haynes R, Paciotti G. CYT6091 (Aurimune): a colloidal gold-based tumortargated nanomedicine. Nanomed 2006; 2(4): 273-74.

16. AurimuneTM (CYT-6091). www.cytimune.com (Accessed on 24 May 2010).

17. Taxol ${ }^{\circledR}$ (Paclitaxel) injection (www.rxlist.com/taxoldrug.htm) (Accessed on 24 May 2010).

18. Huff TB, Tong L, Zhao Y, Hansen MN, Cheng JiXin, Wei A. Hyperthermic effects of gold nanorods on tumor cells. Nanomed 2007; 2:125-32

19. Liao H, Nehl CL, Hafner JH. Biomedical applications of plasmon resonant metal nanoparticles. Nanomed 2006; 1: 201-208 (doi:10.2217/17435889.1.2. 201). 